\title{
Die Energiewende in Europa
}

G. Brauner OVE

Online publiziert am 5. April 2013

(C) Springer Verlag Wien 2013

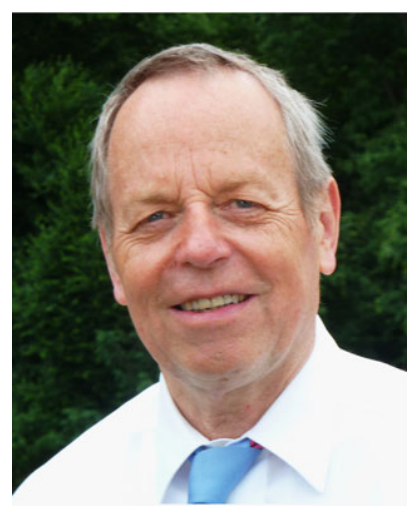

Em. O. Univ.-Prof. Dr. Günther Brauner
In dieser Ausgabe der e\&i finden Sie, sehr geehrte Leserinnen und Leser, den zweiten Teil der Beiträge eines Workshops zur "Energiewende", der im November 2013 in Wien stattfand. Die hier vorliegenden Beiträge thematisieren die Energiewende in Deutschland und in Europa, insbesondere in ihren Auswirkungen auf die Infrastrukturen. Der Workshop wurde vom World Energy Council in Kooperation mit dem OVE, Oesterreichs Energie, dem Klima- und Energiefonds sowie der TU Wien veranstaltet.

Die Auswirkungen der Energiewende in Deutschland wurde mit einer Expertengruppe des VDE in der Studie "Erneuerbare Energie braucht flexible Kraftwerke - Szenarien bis 2020" eingehend untersucht. Der Blick nach Europa in einen längerfristigen Zeitraum bis zum Jahr 2040 findet sich in der EUREL-Studie "Power Vision 2040". Beide Studien setzen voraus, dass der Elektrizitätsbedarf in Zukunft trotz Maßnahmen zur Effizienzsteigerung eher zunehmen wird. Einerseits besteht die europäische Energiestrategie darin, fossile Energieanwendungen durch erneuerbare Elektrizität aus Wasser- kraft, Windenergie und Photovoltaik zu substituieren. Andererseits werden die Effizienzpotenziale durch neue Anwendungen, insbesondere mit Wärmepumpen, Elektromobilität, Automatisierung und IKT, mehr als ausgeglichen, so dass eher ein leichter Bedarfsanstieg zu erwarten ist.

In Deutschland wird der Ausbau der erneuerbaren Energiequellen bis 2020 bewirken, dass die Netzspitzenlast zeitweilig allein aus Windenergie und Photovoltaik gedeckt werden kann. Die Pumpspeicherkapazitäten werden in diesem Zeitraum zwar verdoppelt, aber ihre relative Leistung im Verhältnis zur installierten regenerativen Energie wird sich halbieren. Flexible thermische Kraftwerke stellen daher bis 2020 die einzige verfügbare Lösung zur Bereitstellung von Ausgleichsenergie dar. Der Vorrang der erneuerbaren Energie wird aber bewirken, dass diese doppelt so häufig an- und abgefahren werden müssen und bei niedrigen Volllaststunden die Fähigkeit zu großen Gradienten, geringer Mindestlast und ausreichend hohe Wirkungsgrade, insbesondere in Teillast, aufweisen sollten.

Im Zeitraum nach 2020 sind zeitweilig hohe regenerative Überschusserzeugungen zu erwarten, und es müssen Möglichkeiten zur am Dargebot orientierten Bedarfssteuerung oder zur Umwandlung "Power-to-Gas" und "Gas-to-Power" geschaffen werden. Die thermischen Kraftwerke können dann weiterhin zum Ausgleich der Residuallast eingesetzt werden und zählen dann wegen des geschlossenen Kohlenstoffkreislaufs zu den regenerativen Anlagen. Dies sind allerdings langfristige Szenarien, und es ist noch viel in Forschung und Technologie zu unternehmen, um diese Ziele zu erreichen.

Brauner, Günther, Em. O. Univ.-Prof. Dr., Institut für Energiesysteme und Elektrische Antriebe, Technische Universität Wien, Wien, Österreich

(E-Mail: guenther.brauner+e373@tuwien.ac.at) 